\title{
Cross Layer Techniques used for Improving TCP Performance in Wireless Networks: A Proposed Technique
}

\author{
Ami Vashi \\ D.J Sanghvi College of \\ Engineering \\ Vile Parle
}

\author{
Akshay Pawar \\ D.J Sanghvi College of \\ Engineering \\ Vile Parle
}

\author{
Aruna Gawade \\ D.J Sanghvi College of \\ Engineering \\ Vile Parle
}

\begin{abstract}
Transmission Control Protocol (TCP) running in the Transport Layer of layered architecture models like OSI, has been struggling with poor performance in wireless networks. Various probable solutions have been established by researchers worldwide. One branch of solutions uses the Cross Layer Design for improving TCP performance. Cross Layer Design violates the layered architecture norms of strictly discrete layers, and is used to improve TCP performance in wireless technology. In this paper, we talk about two cross layer techniques that have been established by researchers over past few years, employed to improve TCP performance. We compare, contrast and judge these techniques and propose our own technique for the same.
\end{abstract}

\section{Keywords}

Cross Layer Design, Layered Architecture, Transmission Control Protocol, Congestion, Explicit Congestion Notice, Multi-hop environment, Data Packets.

\section{INTRODUCTION}

\subsection{Disadvantages of Layered Architecture}

As wireless communications become the common mode of data transfer, the layered model is beginning to show shortcomings.

\section{- Invariant nature of wireless networks}

In most wire-line systems, the channel models and physical architectures are well-understood and generally remain invariant. As such, the communication stack is optimally designed for the channel, and applications are designed to remain within the capability of the system. In wireless communications, the random time-varying nature of the channel inherently leads to varying performance of the communication system. This randomness can affect all the layers, and has been shown to cause degradation of system performance.

\section{- Multi hop wireless networks}

Multi-hop wireless networks impose new challenges such as the varying nature of the signal strength, higher bit-error rates, dynamic variations in channel quality, fading effects, interference problems, mobility, shared and contention based MAC, Multi-hop transmission and path selection at network layer needs some degree of interaction amongst different layers so that to optimized the overall network performance. Layered architecture doesn't allow intensive interaction among layers that multi-hop networks demand.

\section{CROSS LAYER DESIGN}

Protocol design by the violation of reference layered communication architecture is cross layered design with respect to the particular layered architecture.[1]

\subsection{Definition}

Cross Layered Design (CLD) with respect to a reference layer architecture is the design of algorithms and protocols that allow interlayer communication according to the need of the application.[2]

It avoids the water-fall like approach of the layered architecture to create a dynamic working and feedback environment. Cross Layer design is mainly used to overcome the shortcomings of the layered architecture in wireless networks. The following is a diagrammatic illustration of Cross Layered Design used in a layered model.

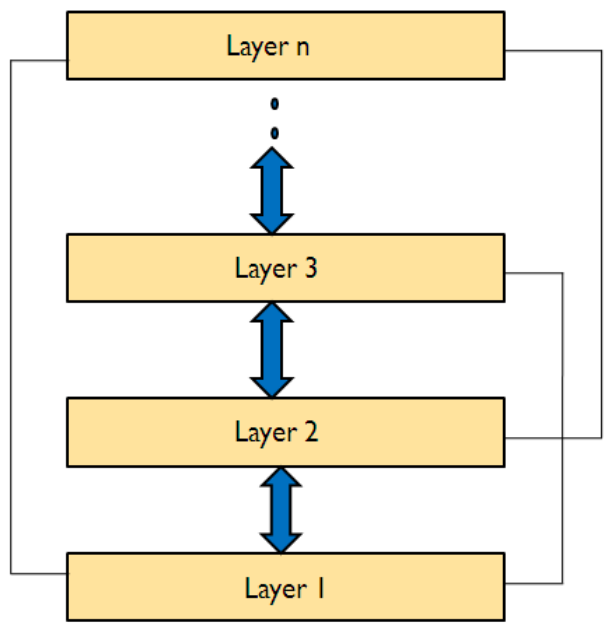

Fig 1 Cross Layer Design diagrammatic representation

\subsection{Shortcoming of the TCP Protocol in the Layered Architecture}

The TCP protocol runs in layer 4 of the OSI model. For wired networks, it successfully reduces loss of packets by imposing various congestion control policies. In wireless networks however, it is observed that the congestion policies do not always help in reducing the packet loss. This is because the packet loss in wireless environment can be due to reasons other than congestion, for instance physical interference or channel fading. However, TCP protocol is programmed to assume that the packet loss is due to congestion and hence, 
layered architecture fails to deal with packet losses due to reasons other than congestion.[3]

\section{ECN BIT APPROACH}

A solution to the above mentioned problem can only be suggested by informing the TCP protocol in layer 4 about the cause of packet loss. This information is available at the physical layer (layer 1), which is inaccessible to the layer 4 in layered architecture.

A violation of the layered architecture norms, by crossing the layer boundaries and enabling communication between layer 4 and the physical layer must be employed. In short, the concept of cross layer design must be used.

The physical layer has a bit named 'Explicit Congestion Notice' (ECN), which determines the reason for the packet loss.[3]

\subsection{Working of ECN Bit Approach}

TCP supports ECN using three flags in the TCP header. The first one is the Nonce Sum (NS), is used to protect against accidental or malicious hiding of marked packets from the TCP sender. The other two bits are used to echo back the congestion indication which means it indicates to the sender that it should reduce the amount of information being sent and to acknowledge that the congestion-indication echoing was received. These are the ECN-Echo (ECE) and Congestion Window Reduced (CWR) bits.

For ECN to be used in a particular wireless network environment, it needs to be negotiated at the time of connection establishment. This can be done in the SYN and SYN-ACK segments of the header.

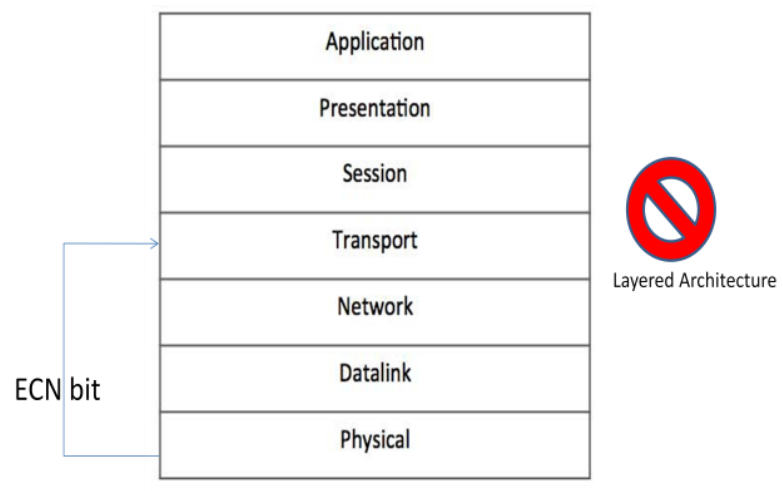

Fig 2. Inter layer communication not allowed in layered architecture

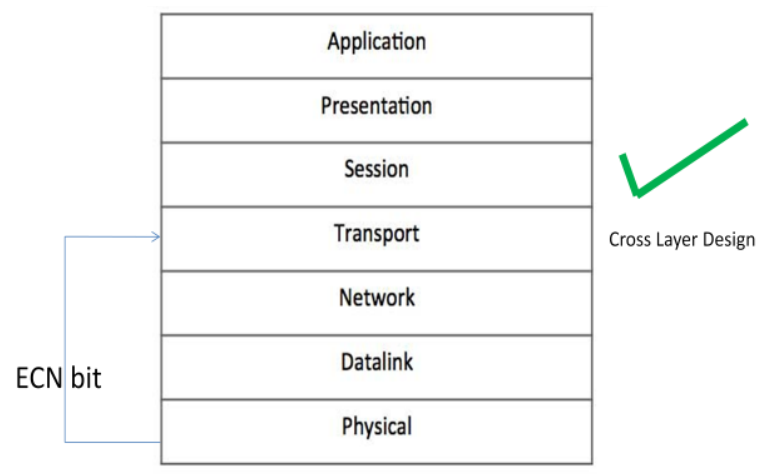

Fig 3 Inter layer communication allowed by Cross Layer Design

\subsection{Actual Implementation}

Various applications have used their unique method of sharing ECN bit and other such information in their CLD architecture.

- Sudame et al use ICMP (Internet Control Message Protocol) messages for propagating lower layer event information to a special handler at socket layer. The adaptation is defined by the application layer.[5]

- MobileMan, an application based on CLD, proposes creation of a new Network Status entity which is used for sharing network information with all the protocol layers. Protocols need to be changed to use this network information.[10]

- Carneiro et al propose a Cross Layer Manager which contains management algorithms.

This manager interacts with the protocol stack for cross layer adaptation.[9]

- Cross Layer Signaling Shortcuts (CLASS) proposes direct interaction between the layers for cross layer adaptation.[6]

- Interlayer Signaling Pipe uses the packet headers to pass adaptation information to lower layers. The layers read the information in the header and adapt accordingly.[7]

- Mehta et al propose user-space implementation for Receiver Window Control. The adaptation is done in user-space and operating system APIs are used for adapting the transport protocol.[8]

In our opinion, since ECN bit access is required by no other layers above the Transport Layer, usage of UDP socket would be advisable as socket communication is quick, and wouldn't hinder the ongoing payload transfer through the Service Access Point (SAP)

\section{MULTI-HOP HIGHER PRIORITY APPROACH}

\subsection{Overview}

In wireless networks, whenever a node is at a large distance from its destination, the packet is required to make multiple hops during transit. This makes it increases the round trip time (RTT) as it has to deal with multiple relay nodes where it must compete with packets of the nearby nodes. This delay largely deteriorates the performance of the TCP protocol. Secondly, the resulting inconsistency in arrival of packets due to their varying distances from the destination further deteriorates the TCP performance. Every packet irrespective of its distance from the destination is given the same bandwidth, in short same priority in the traditional way. Hence packets reaching the destination have more percentage of packets which are from the nearer nodes. This causes a large amount of packet loss for the farther nodes. The imbalance that is created in the network creates unfair background and makes the TCP a weaker protocol in the wireless networks.[4]

The solution to this problem suggested in is FEBA- Fair endto-end bandwidth allocation.[2] FEBA is an algorithm that works towards eliminating the issue of packet loss with an improved outlook and efficiency. It goes hand in hand with the cross layer design architecture. It is basically a distributed algorithm which attempts to solve the glitch of unfair medium access for various traffic flows.

To alleviate the unfairness for farther nodes (higher number of hops), the following steps are performed. It allocates four parameters to every packet: $(\mathrm{s}, \mathrm{d}, \mathrm{n}, \mathrm{p})$ where $\mathrm{s}$ stands for source 
node, $\mathrm{d}$ stands for destination node, $\mathrm{n}$ stands number of hops and $\mathrm{p}$ stands for the priority given to the packet. The priority is computed by the following formula:

\section{Flow Priority $=$ Packet Priority $\times$ Distance to Destination}

After computing the flow priority the packet is appended to a queue of packets at a particular node in its transit path. The packets are dispatched according to their priorities. Packets with equal priorities are sent by 'first in first out' manner. When a packet reaches the next node, then FEBA allocates bandwidth (priority) to the packet in accordance with the number of hops the packet has had so far.

Figure 4 illustrates an elementary example:

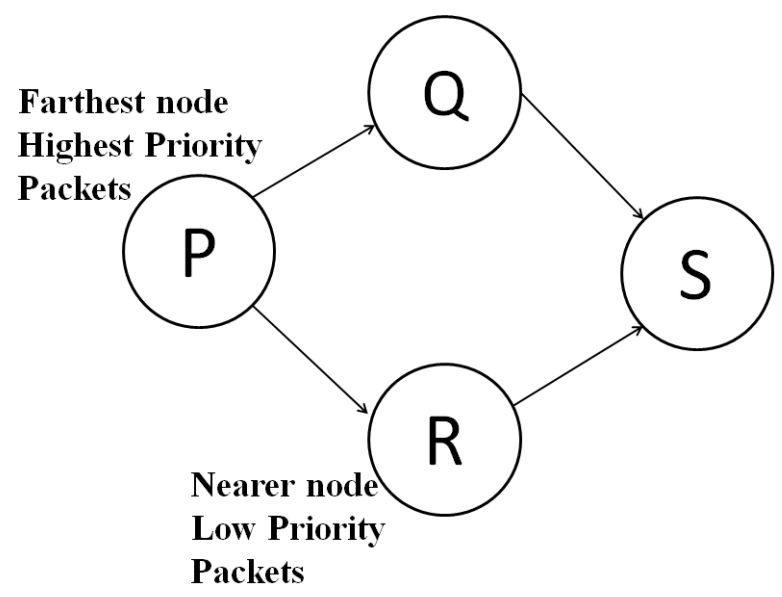

Fig 4 Example illustration MHHP

\subsection{Working}

Here node $\mathrm{R}$ being the nearer node to the destination, gives higher priority to the packets coming from $\mathrm{P}$ over its own packets. The flow priority value computed using the above mentioned formula will always be greater for the farther node. In this way, the unfairness created by larger distance is nullified by giving higher priority to the farther node packets.

Wireless networks are generally ad hoc, hence they are decentralized. There is no central control for the transporting of packets through the network. Therefore all packets must be passed from node to node (router).

In order for a router to efficiently allocate bandwidth based on the FEBA algorithm, it is necessary to make accurate changes in the QoS (Quality of Service) settings of the given router. The router must be given proper instructions about the priority of the packets for wireless transmissions. This ensures that the router handles the bandwidth allocation in a fair and balanced manner thus leading to less packet loss and improvement in the overall performance of the system.

As mentioned earlier, this approach uses CLD, where the boundaries of MAC and network layer are crossed to create a platform of shared functionalities. When there are no communications between the MAC layer and the routing layer, the resource allocations taking place in the MAC layer ignore the fact that some packets are experiencing a longer path and they need special treatment. To mitigate the effect of a longer path on TCP performance, packets coming from farther nodes are prioritized over packets from nearer nodes in MHHP. We investigate the idea of prioritizing flows based on the number of hops from the destination. By taking advantage of cross layer design, the routing level information (i.e., the number of hops to destination) is passed to the scheduling module in the MAC layer. Then, the scheduling module uses the number of hops to destination to decide on the priority of packets in each flow. Results show that the cross layer approach in MHHP increases TCP throughput and stabilizes the fluctuations of RTT.

\begin{tabular}{|c|}
\hline Application \\
\hline Presentation \\
\hline Session \\
\hline Transport \\
\hline MAC + Network \\
\hline DLL \\
\hline Physical \\
\hline
\end{tabular}

Fig 5 MAC and Network Layer crossed

\section{COMPARISON OF ECN AND MHHP}

Both the approaches have great potential of improving TCP performance of wireless networks. The concept of CLD is exploited very well into the approaches making them significant suggestions in mass of research approaches on TCP performance. However, it is important to make fair comparisons between the two approaches by understanding their merits and demerits.

\subsection{Advantages of ECN Bit Approach}

- $\quad$ Simple and quick implementation: Socket usage for ECN access in transport layer will require an addition of a simple socket program in the existing architecture.

- Availability: Since ECN bit in not involved in intensive processes, it is almost always available for access, hence making it convenient to use it an as indication for dynamic wireless network congestion.

- Accuracy: Being in the physical layer, the congestion notice is immediately updated on the ECN bit making it a reliable source of congestion indication.

ECN bit solves a very crucial problem for wireless networks, that is identifying whether congestion has caused packet loss or not. No other source in the network can be as reliable, quick and be easily accessed as the ECN bit.[4]

\subsection{Disadvantages of ECN Bit Approach}

- ECN bit gives incomplete information about the cause of the packet loss. It only indicates whether congestion is the reason for packet or not. Hence if ECN bit mentions that the reason is not congestion, then the packet loss cause still needs to be diagnosed.

- ECN bit approach doesn't act as a solution but simply a congestion indicator. 


\subsection{Advantages of MHHP Approach}

It gives a thorough solution for improving TCP performance in WMNs.

It has been proven in simulations that the TCP is optimized by the use of the algorithms and formulae illustrated in the approach.

\subsection{Judgement}

From the above comparisons it is evident that the approach II has a better outcome, especially in terms of the solutions it provides. The fact that makes it stronger is its simulations provided in the base paper which show a significant optimization of the TCP protocol by the use of MHHP

\section{CONGESTION OR PRIORITY - A NEW PROPOSED APROACH}

'Congestion or Priority' is an approach derived by combining the two approaches discussed in this paper. The basic effort to combine the advantages of the two is apparent in this new approach.

\subsection{Working}

Packet loss and delay when detected, the system may apply the 'Congestion or Priority' approach. Firstly, the ECN bit approach is employed. The ECN approach acts more like a flag for congestion. Here the ECN bit from the physical layer is accessed by the transport layer by using the CLD technique. The CLD technique used here is socket program. The socket is easily implementable to the current system without adding complexity. If congestion is indicated, a congestion control policy is applied by the TCP protocol.

If the congestion is not indicated as the reason for packet loss, then the approach II is applied. Here all the priority algorithms are run and the RTT is minimized to its lower limit. This approach acts more like a solution to the problem but doesn't itself detect the cause. Hence it is sensible to keep approach I serially former to this approach, so that the problem indication is made in advance. Once the necessary algorithms are applied the packet loss and delay is rechecked, if it still exists the entire cycle is repeated, else the process is ended and the system can resume for normal functioning.

In a nutshell, the approaches are being serially put together and their combination is woven in such a way that the problem detection and solution occurs sequentially with good efficiency.

The Flowchart below illustrates the model implementation of the 'Congestion or Priority' approach in a logical order.

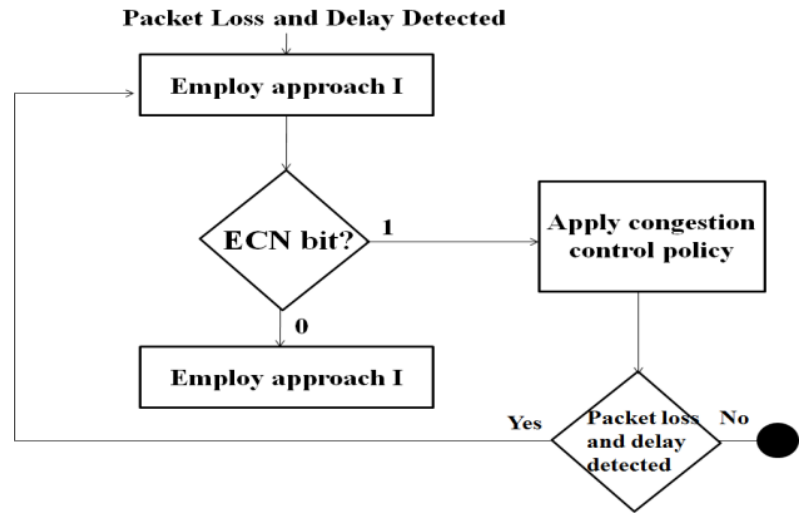

Fig 6 Flowchart illustrating new approach

\section{CONCLUSIONS}

Cross Layer Techniques have been under extensive research for the past decade. It is therefore crucial to study various proposed techniques, compare and contrast them and make a personal judgements on the basis of their credibility. In this paper, we studied two approaches established by two base papers mentioned in the references. These approaches were chosen purely on the basis of their credibility and the chance to make substantial improvements. With logical comparisons of their merits and demerits, we have established on own approach called the 'Congestion or Priority'. It is an approach which tries to extract the merits of the two approaches and make TCP optimization more efficient and apparent. With exponential growth in the users, it is now extremely important to implement measures to manage this traffic. The future scope of cross layer design shows great promise. The flexibility provided by the cross layer techniques aims to provide customization to the users. Corporate intranets will benefit greatly in the future with the help of these techniques. A broad spectrum of possibilities have opened up due to Cross Layer Design.

\section{ACKNOWLEDGEMENTS}

We thank our college, DJ Sanghvi College of Engineering and the department of computer engineering for their constant support in terms of the resources provided, and some valuable advise that helped us throughout our research.

\section{REFERENCES}

[1] Srivastava V, Motani M. Cross-layer design: a survey and the road ahead. IEEE Communications Magazine 2005;

[2] Dzmitry Kliazovich and Fabrizio Granelli"Cross Layer Designs in WLAN systems"in University of Luxemburg 6 rue Condenhove kalergi,L-1359 Luxemburg.DISIUniversity of Trento via Sommarive14,I-38050 Trento,Italy., 2011

[3] Jeffrey Mehlman" Cross-Layer Design: A Case for Standardization"in Electrical Engineering Department, Stanford University Stanford, California 94305 jmehlman at stanford dot edu

[4] Nasim Arianpoo, Paria Jokar, Victor C.M. Leung Department of Electrical and Computer Engineering, The University of British Columbia, Vancouver, BC, Canada V6T 1Z4, "Enhancing TCP Performance in Wireless Mesh Networks by Cross Layer Design", Workshop on Computing, Networking and Communications, 2011.

[5] P. Sudame and B. R. Badrinath. On Providing Support for Protocol Adaptation in Mobile Networks. Mobile Networks and Applications, 6(1):43-55, 2001.

[6] QiWang and M.A. Abu-Rgheff. Cross-layer Signalling for Next-GenerationWireless Systems. In Wireless Communications and Networking (WCNC), volume 2, pages 1084-1089. IEEE, March 2003.

[7] Gang Wu, Yong Bai, Jie Lai, and A. Ogielski. Interactions between TCP and RLP in Wireless Internet. In IEEE GLOBECOM, volume 1B, pages 661-666, Rio de Janeiro, Brazil, December 1999. IEEE.

[8] P. Mehra, A. Zakhor, and C. Vleeschouwer. ReceiverDriven Bandwidth Sharing for TCP. In IEEE INFOCOM, SF, USA, April 2003.

[9] G. Carneiro, J. Ruela, and M. Ricardo. Cross Layer Design in 4G Wireless Terminals. IEEE Wireless Communications, 11(2):7-13, April 2004. 